\title{
International symposium on somatostatin
}

\author{
James A. BARRowman, MB, FRCP (LOND), FRCPC, PhD
}

\section{$\mathrm{T}$} HE ISOLATION, SEQUENCING AND SYNTHESIS OF THE CYCLIC tetradecapeptide, somatostatin, was achieved in 1973 by Brazeau and his colleagues. This discovery has been followed by a huge amount of research in the fields of molecular biology, biochemistry and experimental medicine, recognizing that this compound and its more stable analogues might play a role in the management of a wide spectrum of disease. Major medical specialties in which somatostatin analogues have evident potential include endocrinology, oncology and gastroenterology, though considerable interest in the use of these compounds in neurological disorders is also emerging. The great susceptibility to proteolytic degradation of native somatostatin 14 and 28 , the two major biological forms of the peptide, is reflected in their brief half-lives of 1 or 2 mins. The development of a long acting analogue, SMS 201-995 (Octreotide; Sandostatin; Sandoz Inc), a form resistant to enzymatic degradation, was a necessary undertaking to allow investigation of the peptide as a therapeutic agent. Fedorak, in the May 1989 issue of The Canadian Journal of Gastroenterology, reviewed the clinical potential of this derivative in the management of various gastrointestinal disorders. The present report follows up on that review, highlighting new information about somatostatin and its analogues in the science and clinical practice of gastroenterology, as presented at the recent international symposium on somatostatin in Montreal this summer.

DrJ.A. Barrowman, Faculty of Medicine, Memorial University of Newfoundland, St John's, Newfoundland A1B 3V6
The symposium, which dealt with all aspects of somatostatin biology, included new information on somatostatin gene regulation, biosynthesis and processing of the peptide and its physiological and therapeutic roles in the control of growth hormone and pancreatic islet hormone secretion and in various aspects of central nervous system function. Considerable progress has been made in the isolation and characterization of receptors for somatostatin in various tissues and in understanding the intracellular message systems activated or inhibited by the interaction of the peptide with its receptors. Of particular interest are the neoplasms which express somatostatin receptors affording possible therapeutic strategies for control of growth of these tumours.

Somatostatin is found in high concentrations in the gastric mucosa, particularly in the D cells, which release the peptide in response to feeding. It acts to inhibit gastric acid secretion by paracrine and endocrine actions on $G$ cells and parietal cells. The inhibition of gastric acid secretion by hormones such as neurotensin may be mediated through somatostatin. Release of somatostatin from D cells is partly a response to gastric luminal acidification which thus leads to a paracrine restraint on hydrochloric acid secretion. The release of somatostatin is also regulated by cholinergic neurone activity which inhibits the release of the peptide and noncholinergic (bombesin/gastrin releasing peptide) neurones which stimulate release of somatostatin from D cells. These two opposing mechanisms can be revealed by selective blockade. A balance between the two exists; during maximal activation of interneurones the influence of the cholinergic nerves predominates, so that somatostatin release is inhibited. Cholinergic 
(vagal) stimulation of gastric acid secretion is thus the result of direct stimulation of the parietal cell and an inhibition of the somatostatin restraint mechanism (Makhlouf and Schubert). However, there is evidently great complexity in the effects of cholinergic agents on the D cell since it appears that these substances have both stimulative and inhibitory actions on somatostatin release, acting through different intracellular messenger systems (Chiba, Park and Yamada).

Both natural forms of somatostatin (SS), ie, SS-14 and SS-28, inhibit exocrine pancreatic secretion in vivo but neither has any effect on in vitro preparations of acinar cells. Studies by Debas et al suggest that the inhibition caused by somatostatin in vivo requires neuronal transmission but involves neither cholinergic nor adrenergic neurones. It is possible that somatostatin exerts its effect through inhibitory peptidergic neurones. This raises the question of what function is served by the somatostatin receptors which are demonstrated to be present on acinar cell membranes and which have been extensively purified.

Somatostatin has been reported to be present in pancreatic juice, but a recent investigation by Morriset and his colleagues has identified a unique protease in rat pancreatic juice which degrades somatostatin and which, by degrading the radiolabelled somatostatin used in the assay, results in false detection of the peptide in pancreatic juice. It is of interest that this 'somatostatinase' is secreted in an inverse relationship to other pancreatic enzymes and does not appear to be stored in significant amounts in zymogen granules; it is secreted in pancreatic juice in a 'nonparallel' fashion, as compared with other pancreatic hydrolases.

\section{CLINICAL GASTROENTEROLOGY}

Since receptors for somatostatin and Sandostatin have been demonstrated in a variety of human endocrine and nonendocrine tumours, the development of a ${ }^{123}$-labelled somatostatin analogue by Lamberts et al has allowed imaging and localization of such tumours. Primary and metastatic lesions can be visualized in a large proportion of patients with various tumours such as carcinoids, paragangliomas, neuroblastomas, vipomas and small cell lung cancers. It is also possible to visualize some pheochromocytomas and medullary carcinomas of the thyroid, and it was suggested that if such a tumour produces large amounts of somatostatin this might result in nonvisualization of the tumour by a competitive effect. Whether this is the correct explanation, rather than a lack of receptors on the tumour cells, is not clear, since it was reported that a somatostatinoma could be visualized by this technique. An important point is that if the tumours can be readily visualized by this technique the patients appear to respond well to somatostatin analogues. A further possibility of targeted radiotherapy of such tumours is an exciting one though great care would be needed to shield nontumorous tissues.

Considerable success has been reported in the treatment of neuroendocrine tumours with somatostatin analogues, the most responsive being vipomas and carcinoids. Kvols and Reubi reported results with somatostatin analogue in $66 \mathrm{pa}-$ tients with metastatic carcinoids in whom flushing was controlled in $87 \%$ and diarrhea in $77 \%$; a major reduction in urinary 5-HIAA was observed in $77 \%$. Encouraging results in terms of median survival were also reported. Again it was pointed out that there was a correlation between the density of somatostatin receptors in the tumour tissue and the clinical response to the somatostatin analogue.

In gastrinoma, the somatostatin analogue, Sandostatin, appears to reduce gastrin and gastric acid secretion (Vinik). With regard to tumour mass, where reductions have been reported in other neuroendocrine tumours such as vipomas, there was little evidence of such an effect, at least in patients with very high basal gastrin levels, though somatostatin analogue did arrest growth in some tumours, Gastrinomas producing other hormones besides gastrin, notably adrenocorticotropic hormone, appear to be resistant to somatostatin therapy and exhibit unrestrained tumour progression despite somatostatin therapy.

Tumour growth arrest or regression has occurred frequently enough with somatostatin and its analogues to warrant intensive investigation. The mechanism is not clear and could be multifactorial, including effects on tumour blood supply. There is evidence from in vitro studies of tumour cell lines that somatostatin receptor occupancy leads to antagonism of growth mechanisms in the cell normally mediated by specific protein phosphorylation steps, and the antagonistic effect of somatostatin may involve stimulation of a tyrosine phosphatase (Liebow).

Somatostatin has an antisecretory effect on intestinal fluid and electrolyte transport and inhibits electrogenic anion secretion while stimulating salt and water absorption by the intestine (Gaginella). Its use in various diarrheal states has naturally been explored. The analogue of somatostatin is useful in reversing the secretory diarrhea of vipomas and in correcting the associated hypokalemia. Other agents whose action as secretagogues to the intestine is inhibited by somatostatin include bradykinin, serotonin and prostaglandins. These substances are frequently produced by neuroendocrine tumours. Success has also been reported in the treatment of fluid and electrolyte diarrheal losses in the rare condition of congenital microvillus atrophy of neonates. The diarrhea seen in acquired immune deficiency syndrome (AIDS) patients, due to parasitic infection (eg, microsporidia or cryptosporidia) or to causes as yet not identified as intestinal infections, appears to respond to somatostatin analogue therapy. The human immunodeficiency virus itself can colonize the mucosa and recent studies have detected a three-amino acid homologous sequence in the viral glycoprotein coat and in vasoactive intestinal peptide, suggesting the possibility that the virus may have a direct secretagogue action on the enterocyte.

Many gastroenterological conditions have been treated with somatostatin analogue. These include gastrointestinal and pancreatic external fistulae, chronic pancreatitis, necrotizing acute pancreatitis and short gut syndrome. Diabetic diarrhea and high output ileostomy diarrhea have also been reported to respond to somatostatin. Encouraging results have been reported in terms of closure of external intestinal or pancreatic 
fistulae through reduction of secretion. Gastrointestinal bleeding disorders such as esophageal variceal hemorrhage have also been examined since somatostatin is a powerful splanchnic vasoconstrictor. While encouraging results have been obtained in some investigations of these several conditions, conflicting results are common. Thus there is need for carefully controlled studies to substantiate the enthusiastic claims for efficacy of somatostatin in various situations. Woltering and colleagues reported their results of an examination of the response of post gastrectomy dumping syndrome to Sandostatin in a double-blind randomized study of nine patients. A $40 \%$ decrease in the intensity of symptoms was recorded and substantial decreases in meal-stimulated gastrointestinal peptide blood concentrations occurred. A late, late dumping syndrome at 3 to $4 \mathrm{~h}$ resulting from hypoglycemia could be averted by judicious reduction of somatostatin dose. The patients were subsequently able to adjust their own dose to obtain optimal effect.
Somatostatin and its analogues are clearly of intense clinical interest. The full clinical potential of these compounds is yet to be realized. Some of their properties - for example, inhibition of cell growth and an anti-angiogenic effect - open new vistas in gastroenterology as well as other clinical disciplines; we can continue to anticipate refinements in the use of these compounds in diagnosis and therapy of many disorders of widely differing pathogeneses.

\section{FURTHER READING}

Somatostatin in gastroenterology. Dig Dis Sci 1989; 34(Suppl).

Fedorak RN. Long acting somatostatin analogue: Clinical potential for gastrointestinal disease. Can J Gastroenterol 1989:3:77-81.

Kovls LK, Moertel CG, O'Connell MJ. Treatment of the malignant carcinoid syndrome. N Engl J Med $1986 ; 315: 663-6$. 


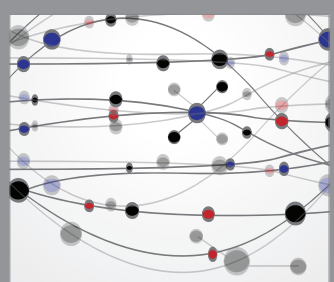

The Scientific World Journal
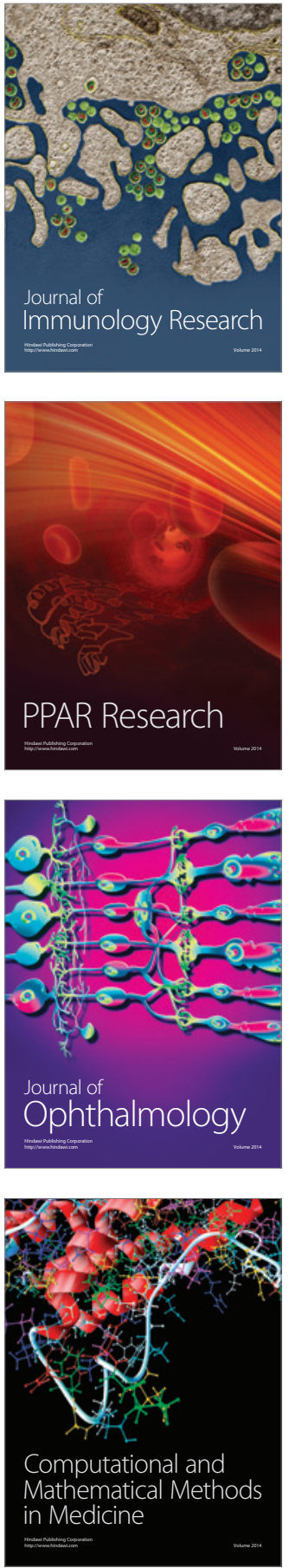

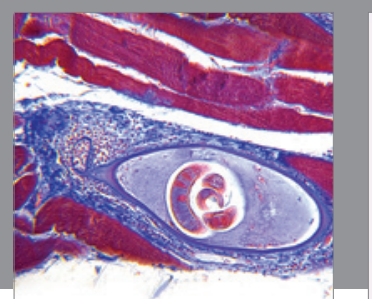

Gastroenterology Research and Practice

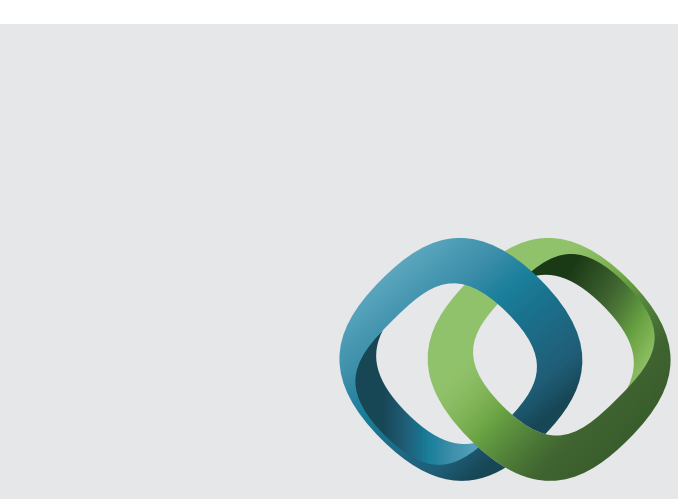

\section{Hindawi}

Submit your manuscripts at

http://www.hindawi.com
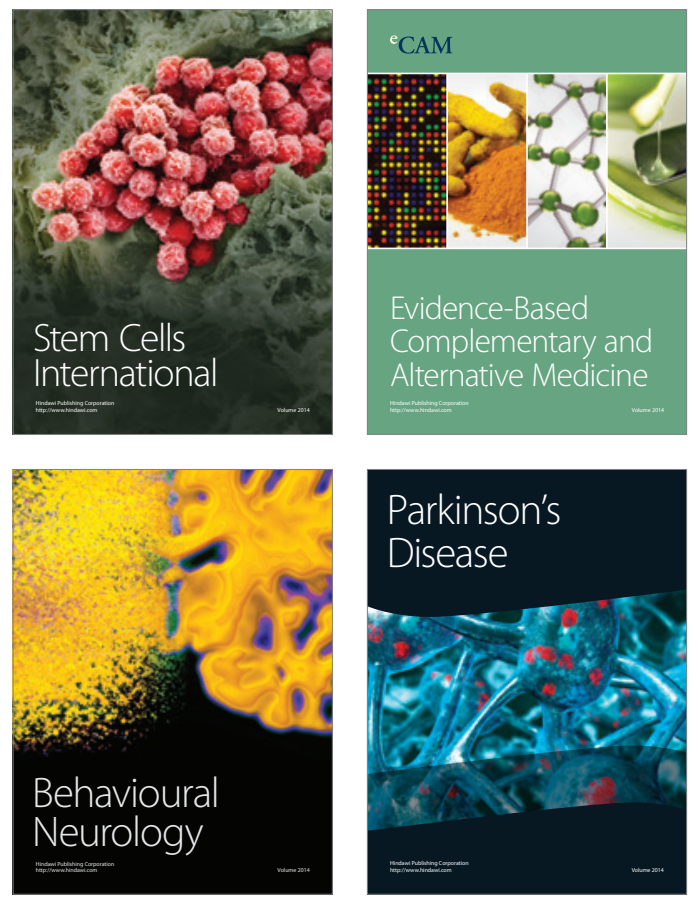
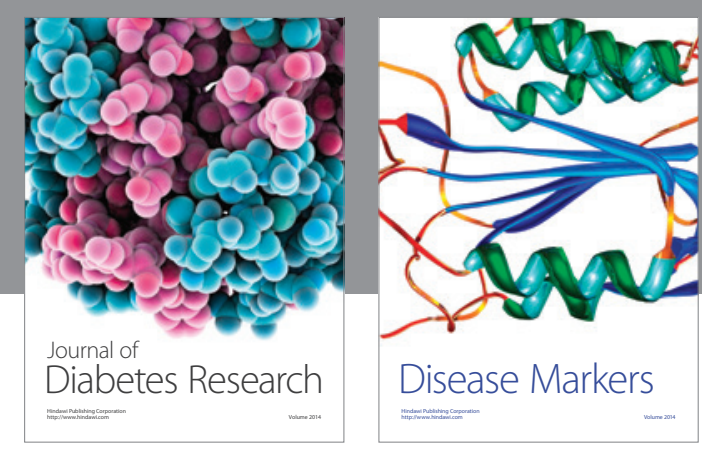

Disease Markers
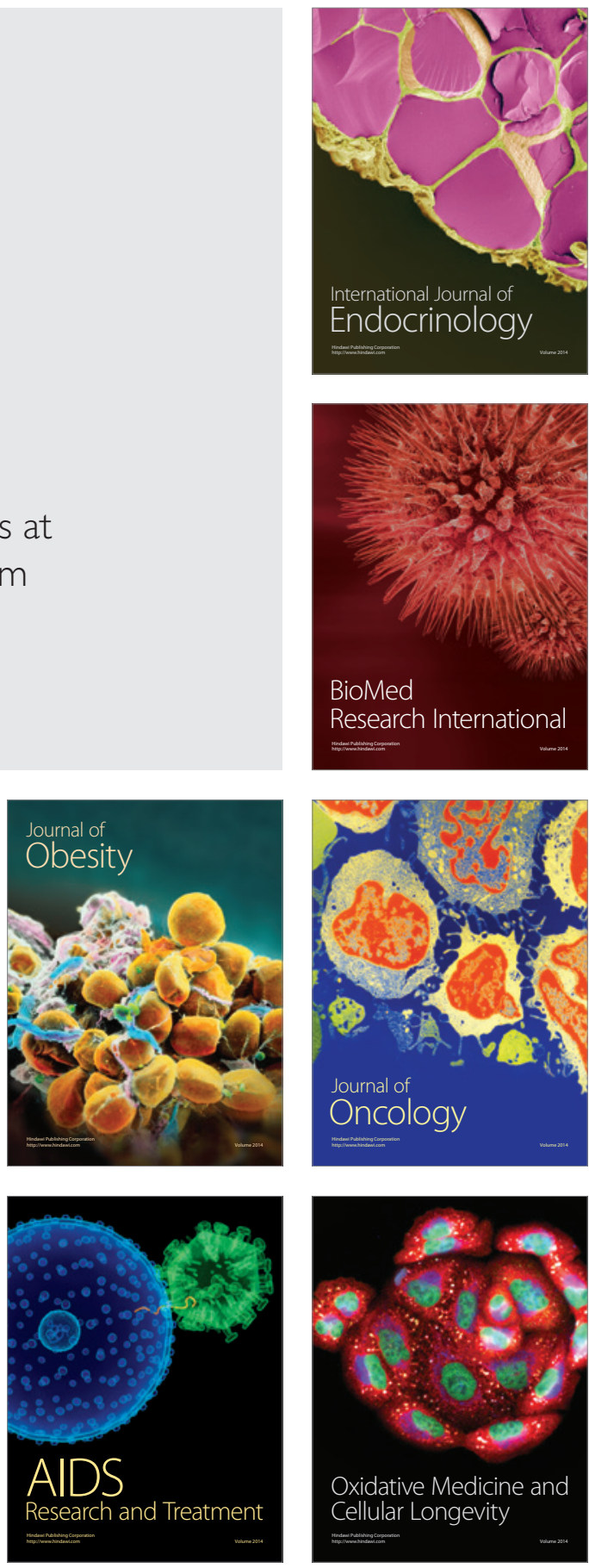